\title{
KIF1B and NF1 are the most frequently mutated genes in paraganglioma and pheochromocytoma tumors
}

\section{Dear Editor,}

Pheochromocytomas and paragangliomas (PPGL) are rare neuroendocrine tumors arising from the adrenal medulla and extra-adrenal paraganglia, respectively. Forty percent are explained by germline mutations in known susceptibility genes. Furthermore, somatic mutations were identified in an additional 30\% of PPGL, mostly in NF1, RET, VHL, MAX and HRAS. However, screening is limited to a few studies mainly using Sanger sequencing (Burnichon et al. 2011, 2012, Crona et al. 2013, Luchetti et al. 2015, Weber et al. 2012). Our aim was to look for the prevalence and nature of somatic mutations using a targeted deep-sequencing approach in patients from a Belgian multicentric PPGL cohort. Targeted Next Generation Sequencing was performed in 74 tumors using a panel including 17 susceptibility genes. Variants considered as damaging by at least 5 prediction programs, or present in COSMIC and considered as damaging by at least 3 prediction programs, were considered to be diseaseassociated mutations. Somatic mutations were identified in $54 \%$ of patients. The most frequently mutated genes were NF1 (20.8\%) and KIF1B (20.4\%). While the high prevalence of somatic mutations in NF1 is in agreement with previous studies, the similarly elevated prevalence of mutations in KIF1B is novel.

Tumor and corresponding blood samples or normal adrenal gland tissue were available for 74 PPGL consecutive patients (55 paraffin-embedded and 19 frozen samples). The mean age at diagnosis was $45.4 \pm 16.2$ years, and $55 \%$ were females. Forty-seven tumors (63\%) were pheochromocytomas (PCCs), and among the 27 paragangliomas (PGLs), 23 (85\%) were located in the head and neck region. Seven (9\%) PPGLs were multifocal, $10(13 \%)$ were recurrent, $6(8 \%)$ were malignant, because of evidence of metastasis, and 7 (9\%) patients had a familial history of PPGL. None of the patients had features suggestive of Multiple Endocrine Neoplasia 2, Von Hippel-Lindau disease or Neurofibromatosis.
Fourteen patients (15\%) harbored a previously identified germline mutation: 9 SDHD, 2 SDHB, 1 SDHA, 1 VHL and 1 MAX. The study was approved by the Comite d'Ethique Hospitalo-Facultaire des Cliniques universitaires SaintLuc-UCL (CEHF).

An Ion AmpliSeq Custom DNA Panel for formalinfixed paraffin-embedded (FFPE) tissues (Life Technologies) was designed to amplify all exons of 17 known PPGL susceptibility genes: EPAS1, FH, HIF1A, HRAS, KIF1B, MAX, NF1, PHD2, RET, SDHA, SDHAF1, SDHAF2, SDHB, SDHC, $S D H D, T M E M 127$ and $V H L$ (primers available upon request). Libraries were prepared according to manufacturers' instructions (Ion Ampliseq Library Preparation, Publication MAN0006735), as more extensively described by Soblet et al. (2017). Each sample yielded two barcoded libraries that together covered the coding regions of all 17 genes. After purification and concentration measurement, emulsion PCR followed by Ion Sphere Particle (ISP) enrichment was performed using the Ion OneTouch 200 Template Kit V2 on a OneTouch 2 System (both from Life Technologies) according to manufacturer's protocol (Ion PGM Template OT2 200 Kit, Publication MAN0007220). Prepared templates were loaded onto an Ion 316 Chip V2, and sequenced using the Ion PGM Sequencing 200 Kit v2 on an Ion PGM (all from Life Technologies; protocol in Publication MAN0007273). The tumoral DNA was sequenced with an Ion Proton to reach a higher coverage.

Sequences, generated by PGM, were aligned into BAM files by the Torrent Server. Aligned sequence (BAM) files were submitted to the Torrent Variant Caller for variant calling (Life Technologies). Variants were annotated, filtered and visualized using Highlander, an in-house bioinformatics framework (Helaers R, Brouillard P, Soblet J, Amyere M, Limaye N \& Vikkula M, unpublished observations; http://sites.uclouvain.be/highlander/). The called variants were retained if they passed the following criteria: (1) absent from germline DNA of the same patient,

Published by Bioscientifica Ltd 
(2) total depth of coverage of $\geq 100$ reads at the variantposition, (3) $\geq 1 \%$ variant-allele frequency and (4) $\geq 5$ mutant reads, with at least one forward and one reverse read. Somatic variants that passed these criteria were evaluated as follows: (1) Synonymous somatic variants were discarded; (2) somatic variants present in $>5$ alleles in Genome of the Netherlands (GoNL) and in $>10$ alleles in 1000 Genomes were discarded; (3) somatic variants present in $>20 \%$ of all the sequenced PPGL tissues were discarded; (4) visual inspection of all remaining somatic variants was performed using the Integrated Genome Viewer (IGV; Broad Institute). Somatic variants within homopolymers, known to be error-prone using this technology, were discarded. Finally, only the somatic variants having a $\mathrm{z}$-score $\geq 5$ were retained. Z-scores were calculated separately for frozen and FFPE tissues, as described by Soblet et al. (2017). Missense somatic mutations were submitted to six programs (Polyphen2, SIFT, Mutation Taster, Mutation Assessor, Functional Analysis though Hidden Markov Models or FATHMM, Likelihood Ratio Test or LRT, all implemented in Highlander) to predict their effect on protein function. The somatic variants predicted as damaging by $\geq 5$ programs or by $\geq 3$ programs and present in the COSMIC database were considered as somatic mutations.

The main results of our study can be summarized as follows. Somatic mutations were identified in 40 patients (54\%), only 8 of which carried a germline mutation

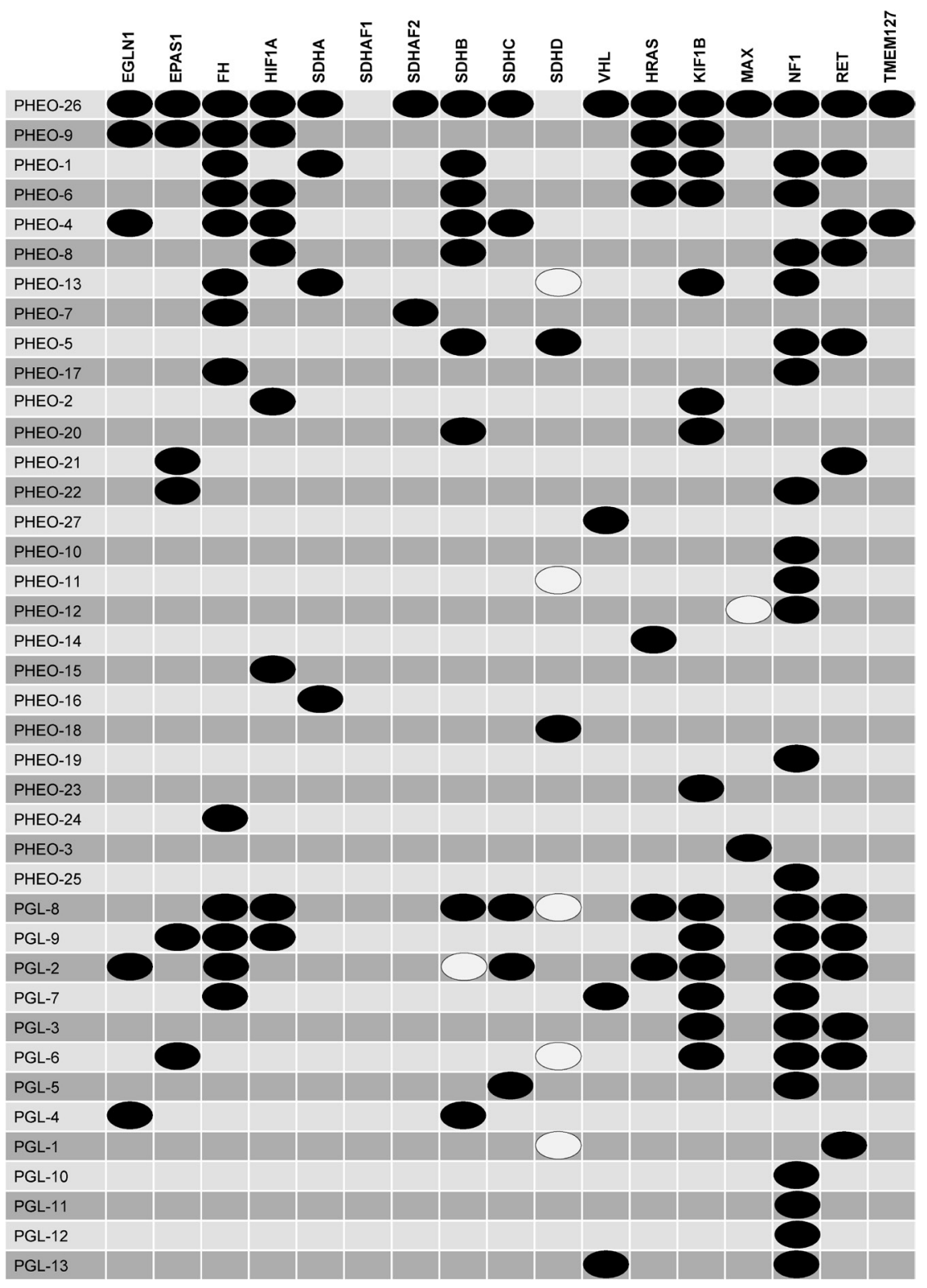

Figure 1

Distribution of mutations in patients. Black dots indicate somatic mutations; light gray dots indicate germline mutations; PHEO indicates adrenal tumor; PGL indicates extra-adrenal paraganglioma. 
(6SDHD, 1 SDHB and $1 M A X)$ (Fig. 1). Using gene expression profiling, PPGLs can be classified in two clusters: Cluster 1 (hypoxia cluster) containing $S D H x-, F H$ - and $V H L$-related tumors, and Cluster 2 (kinase signaling cluster) including MAX-, NF1-, RET- and TMEM127-mutated PPGLs (Burnichon et al. 2011). Mutations were more frequent in genes involved in Cluster 2 than in Cluster 1 (58\% vs $42 \%, P$ value $=0.003$ ). The average number of mutations per patient was $7.9 \pm 19.4$ (Fig. 2A). The most frequently mutated genes were NF1 and KIF1B. Indeed, mutations in these two genes accounted for $21 \%$ and $20 \%$ of the total number of mutations, respectively (Fig. 2B). Furthermore, mutations in NF1 and KIF1B were found in 28\% and 19\% of all patients, respectively (Fig. 2C). In addition, a Burden test, comparing the proportion of variants found in our samples and the ones reported in the ExAC database, could be performed using Highlander. For both genes, the proportion of rare, damaging variants, as defined above, was higher in patients than in $\operatorname{ExAC}(P$ values $<0.001)$. Finally, even though $K I F 1 B$ has a shorter coding region than NF1 (5.4 kb vs $8.5 \mathrm{~kb})$, both genes carried almost the same number of mutations (65 and 66, respectively); the density of mutations is therefore higher in KIF1B than in NF1 (11.9 mutations/kb vs 7.7 mutations/kb).

Somatic mutations in the NF1 and KIF1B genes were most common, followed by $F H$ and RET (18\% and $16 \%$ of patients, respectively). Interestingly, RET mutations were predominantly found in PGLs compared to PCCs $(54 \%$ vs $18 \%, P$ value $=0.02)$. No significant difference in the proportion of NF1 and KIF1B mutations was observed between PGLs and PCCs (NF1: $61 \%$ vs $48 \%, P=0.43 ; K I F 1 B$ : $46 \%$ vs $30 \%, P=0.30)$. All other tested genes accounted for less than $10 \%$ of mutations. Notably, the types and proportions of somatic mutations were comparable between the 55 FFPE and 19 frozen tumors. Overall, mutations were found in 53\% of FFPE tissues and in 58\% of frozen samples $(P$ value $=0.69)$, and the proportions of mutations in NF1 (16\% vs 23\%, $P$ value=0.13) and in $K I F 1 B(18 \%$ vs $23 \%, P$ value $=0.29)$ were similar in both subsets.

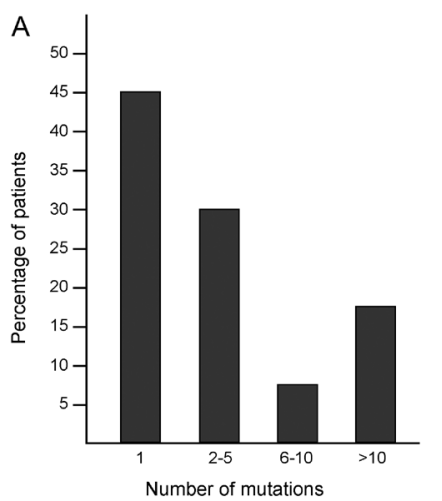

B
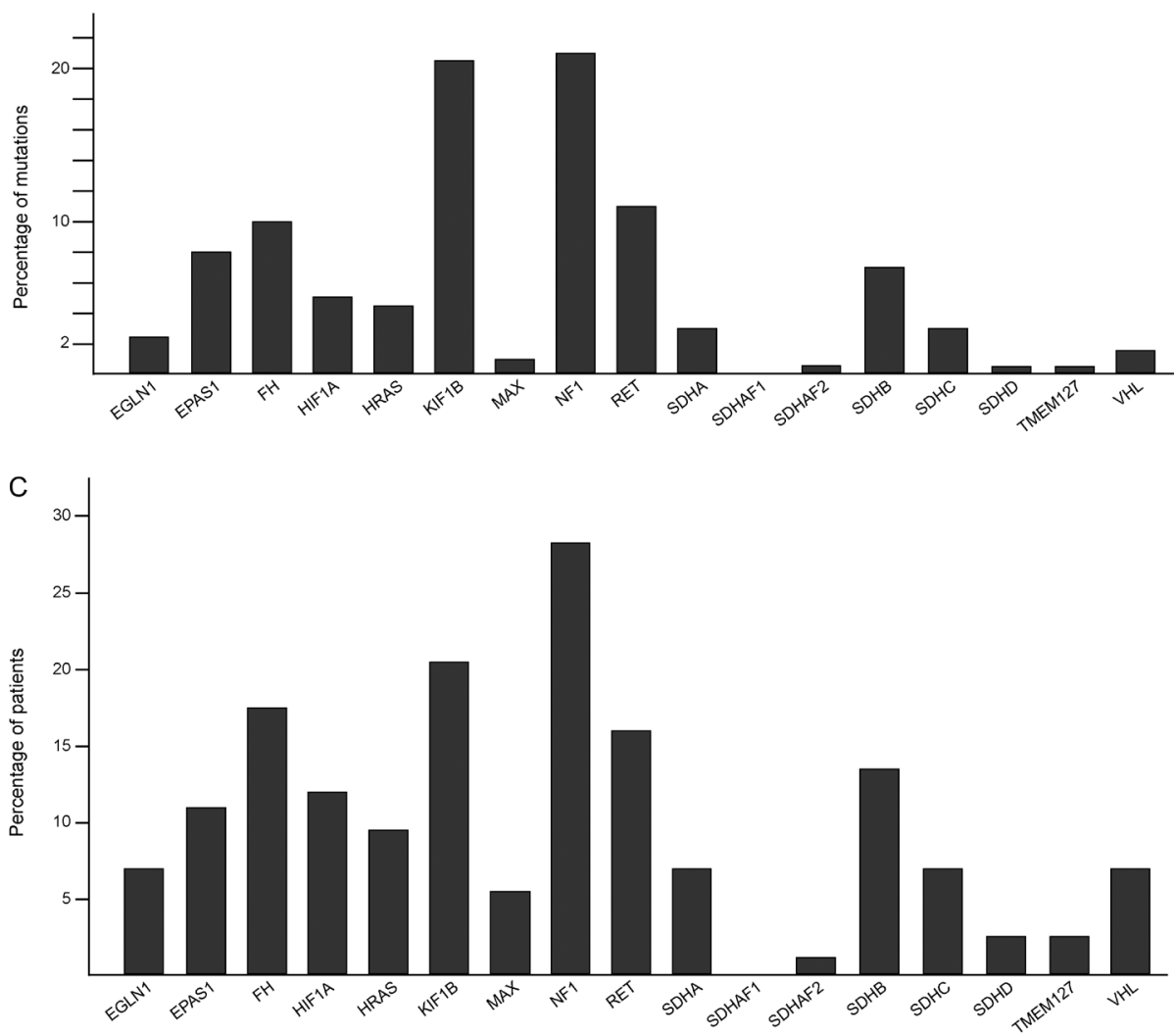

Figure 2

Proportions of identified mutations and mutated patients. (A) Proportion of patients with different numbers of somatic mutations; (B) proportion of mutations in each sequenced gene relative to total number of mutations; (C) proportion of patients carrying mutations per sequenced gene, for example, $20 \%$ of patients carried at least one mutation in KIF1B. 
In conclusion, using a panel including 17 susceptibility genes, we documented the presence of somatic mutations in over $50 \%$ of PPGL tumors. We confirmed the high frequency of NF1 somatic mutations and identified KIF1B as the second most frequently mutated gene in PPGL tissues. Welander and coworkers reported a substantially lower frequency of KIF1B mutations (4\%) (Welander et al. 2014). This is likely due to the variant-allele frequency threshold set at $10 \%$ in the work of Welander and coworkers, while we looked at variants present in $\geq 1 \%$ of the reads. Nowadays, the $1 \%$ variant-allele frequency threshold is commonly used in somatic mutation analyses (Limaye et al. 2015). Finally, RET mutations were found in more than half of mutated patients with head and neck PGL.

Interestingly, we identified a minority of samples with a high number of somatic mutations, which may reflect perturbations in DNA damage repair, and, as such, deserve a more in-depth investigation (Alexandrov et al. 2013). Hypermutated tumors have never been reported in PPGL, but are well described in other tumors, such as adrenocortical carcinomas (Assie et al. 2014). Genetic profiling of PPGLs may therefore help to individualize management and therapy of these tumors.

\section{Lucie Evenepoel ${ }^{1,2}$ Raphaël Helaers ${ }^{2}$ Laurent Vroonen ${ }^{3}$ Selda Aydin ${ }^{4}$ Marc Hamoir ${ }^{5}$ Dominique Maiter ${ }^{6}$ Miikka Vikkula $^{2}$ Alexandre Persu1,7}

${ }^{1}$ Pole of Cardiovascular Research, Institut de Recherche Expérimentale et Clinique, Université catholique de Louvain, Brussels, Belgium

${ }^{2}$ Human Molecular Genetics, de Duve Institute, Université catholique de Louvain, Brussels, Belgium

${ }^{3}$ Department of Endocrinology, Centre Hospitalier Universitaire de Liège, University of Liège, Domaine Universitaire du Sart-Tilman, Liège, Belgium ${ }^{4}$ Pathology Department, Cliniques Universitaires Saint-Luc, Université Catholique de Louvain, Brussels, Belgium ${ }^{5}$ Otolaryngology Department, Cliniques Universitaires SaintLuc, Université Catholique de Louvain, Brussels, Belgium

${ }^{6}$ Endocrinology Department, Cliniques Universitaires SaintLuc, Université Catholique de Louvain, Brussels, Belgium
${ }^{7}$ Cardiology Department, Cliniques Universitaires Saint-Luc, Université Catholique de Louvain, Brussels, Belgium

(Correspondence should be addressed to L Evenepoel or A Persu; email: lucie.evenepoel@uclouvain.be or alexandre.persu@uclouvain.be)

Declaration of interest

The authors declare that there is no conflict of interest that could be perceived as prejudicing the impartiality of this article.

\section{Funding}

This study was supported by a grant 'F.R.S.-FNRS-Télévie' and a grant FRSM 3.4510.11 (to A P) and the Foundation against Cancer, Belgium (to M V). L E was supported by a F.R.S.-FNRS-Télévie fellowship.

\section{Acknowledgements}

We would like to thank Dr Nisha Limaye (de Duve Institute, UCL, Brussels, Belgium) for her permanent help and advice. We are also grateful to Francesca Severino (Cliniques Universitaires Saint-Luc, UCL, Brussels, Belgium) for her precious help in maintenance and improvement of the UCL PPGL database.

\section{References}

Alexandrov LB, Nik-Zainal S, Wedge DC, Aparicio SA, Behjati S, Biankin AV, Bignell GR, Bolli N, Borg A, Borresen-Dale AL, et al. 2013 Signatures of mutational processes in human cancer. Nature $\mathbf{5 0 0}$ 415-421. (doi:10.1038/nature12477)

Assie G, Letouze E, Fassnacht M, Jouinot A, Luscap W, Barreau O, Omeiri H, Rodriguez S, Perlemoine K, Rene-Corail F, et al. 2014 Integrated genomic characterization of adrenocortical carcinoma. Nature Genetics 46 607-612. (doi:10.1038/ng.2953)

Burnichon N, Vescovo L, Amar L, Libe R, de Reynies A, Venisse A Jouanno E, Laurendeau I, Parfait B, Bertherat J, et al. 2011 Integrative genomic analysis reveals somatic mutations in pheochromocytoma and paraganglioma. Human Molecular Genetics 20 3974-3985. (doi:10.1093/hmg/ddr324)

Burnichon N, Buffet A, Parfait B, Letouze E, Laurendeau I, Loriot C, Pasmant E, Abermil N, Valeyrie-Allanore L, Bertherat J, et al. 2012 Somatic NF1 inactivation is a frequent event in sporadic pheochromocytoma. Human Molecular Genetics 21 5397-5405. (doi:10.1093/hmg/dds374)

Crona J, Delgado Verdugo A, Maharjan R, Stalberg P, Granberg D, Hellman P \& Bjorklund P 2013 Somatic mutations in H-RAS in sporadic pheochromocytoma and paraganglioma identified by exome sequencing. Journal of Clinical Endocrinology and Metabolism 98 E1266-E1271. (doi:10.1210/jc.2012-4257)

Limaye N, Kangas J, Mendola A, Godfraind C, Schlogel MJ, Helaers R, Eklund L, Boon LM \& Vikkula M 2015 Somatic activating PIK3CA mutations cause venous malformation. American Journal of Human Genetics 97 914-921. (doi:10.1016/j.ajhg.2015.11.011)

Luchetti A, Walsh D, Rodger F, Clark G, Martin T, Irving R, Sanna M, Yao M, Robledo M, Neumann HP, et al. 2015 Profiling of somatic mutations in phaeochromocytoma and paraganglioma by targeted next generation sequencing analysis. International Journal of Endocrinology 2015138573.
(C) 2017 Society for Endocrinology Printed in Great Britain
Published by Bioscientifica Ltd. 
Soblet J, Kangas J, Natynki M, Mendola A, Helaers R, Uebelhoer M, Kaakinen M, Cordisco M, Dompmartin A, Enjolras O, et al. 2017 Blue Rubber Bleb Nevus (BRBN) syndrome is caused by somatic TEK (TIE2) mutations. Journal of Investigative Dermatology 137 207-216. (doi:10.1016/j.jid.2016.07.034)

Weber A, Hoffmann MM, Neumann HP \& Erlic Z 2012 Somatic mutation analysis of the SDHB, SDHC, SDHD, and RET genes in the clinical assessment of sporadic and hereditary pheochromocytoma. Hormones and Cancer 3 187-192. (doi:10.1007/s12672-012-0113-y) Welander J, Andreasson A, Juhlin CC, Wiseman RW, Backdahl M, Hoog A, Larsson C, Gimm O \& Soderkvist P 2014 Rare germline mutations identified by targeted next-generation sequencing of susceptibility genes in pheochromocytoma and paraganglioma. Journal of Clinical Endocrinology and Metabolism 99 E1352-E1360. (doi:10.1210/jc.2013-4375)

Received in final form 25 April 2017

Accepted 16 May 2017

Accepted Preprint published online 17 May 2017
Published by Bioscientifica Ltd. 